\title{
CYTOCHEMICAL DEMONSTRATION OF CATABOLIC ACTIVITY IN SPERMATOZOA BY THE FORMAZAN TEST
}

\author{
F. HRUDKA \\ Department of Histology and Embryology, Faculty of Veterinary Medicine, \\ Košice, Czechoslovakia \\ (Received 22nd Fuly 1964, revised 3rd November 1964)

\begin{abstract}
Summary. A cytochemical method for the demonstration of endogenous dehydrogenase activity, i.e. as exhibited in the absence of added substrates, is described in spermatozoa of bull, man and rabbit. This activity is confined to the mitochondrial sheath of the flagellum, and decreases both with spontaneous ageing of sperm and under the influence of certain physical and chemical factors. The method can be applied in spermiology for experimental and diagnostic purposes.
\end{abstract}

\section{INTRODUCTION}

While studying specific intracellular dehydrogenases of bull spermatozoa by cytochemical methods, we observed marked dehydrogenase (Nitro BT reductase) activity in living spermatozoa, directed against certain hydrogen acceptors (Hrudka, 1963, 1964). This led us to investigate the possibility of applying the dehydrogenase test for the purpose of assessing the viability of spermatozoa. It is the purpose of this paper (i) to present a detailed description of a cytochemical dehydrogenase test based on the formation of formazan, (ii) to clarify the underlying mechanism and (iii) to outline the advantages of applying that test in the practical evaluation of spermatozoa.

\section{MATERIAL AND METHODS}

Intracellular dehydrogenase activity was investigated in the spermatozoa of bull, rabbit and man. The greatest part of the work was carried out with ejaculated bovine spermatozoa.

\section{General description of the cytochemical reaction}

Before carrying out the reaction the semen sample, the reagents and the chemically clean glass are all adjusted to room temperature. Then a drop of semen and one or two drops of the 'incubating medium' (the amount depending on the actual concentration of spermatozoa in the sample) are gently mixed together on a depression slide, using the edge of a cover glass, then covered with the cover glass and incubated at $37^{\circ} \mathrm{C}$. The use of a depression slide is not absolutely necessary; the reaction can also be carried out on an ordinary slide or watch glass or in a test tube, care being taken, however, at all times to 
prevent the mixture from drying before or during incubation. However, the reaction is carried out most easily on the depression slide, and it also has the advantage of requiring the minimum of semen and of the reagent.

\section{'Incubating medium' and conditions of incubation}

The 'incubating medium' is prepared by dissolving $10 \mathrm{mg}$ Nitro BT $\left(2,2^{\prime}\right.$ di - $p$-nitrophenyl -5,5' - diphenyl-3,3'-dimethoxy-4,4'- biphenyleneditetrazolium chloride) in $10 \mathrm{ml}$ of $0.1 \mathrm{~m}$ phosphate buffer, $\mathrm{pH} 7.5$. This solution can be stored in the refrigerator for up to 3 months. Contamination of the reagent with substances which might reduce Nitro BT or serve as exogenous substrates for sperm must be prevented. The success of the test depends largely upon the quality of the tetrazolium salt. We used mainly the preparation of Nitro BT which has been synthesized by Dr B. Večerek (Prague). With that preparation we obtained the best results at a concentration of $0.5 \mathrm{mg} / 1 \mathrm{ml}$ of 'incubating medium' and an incubation time of $15 \mathrm{~min}$. Using Nitro BT of the Sigma Co, U.S.A., we found it necessary to prolong the incubation period to $60 \mathrm{~min}$.

\section{Preparation of the smears}

On completion of incubation, one or two smears are made from each sample. After they have been air-dried the smears are fixed for $10 \mathrm{~min}$ in formol-saline (one part $40 \%$ formaldehyde to four parts saline), washed for $10 \mathrm{~min}$ in running water and dried again. The smears thus treated can be stored unmounted (in much the same way as blood smears) and then examined by phase-microscopy. If no phase-microscope is available, then contrast staining is recommended. The following procedure may be applied. Formol-fixed and washed smears are rinsed in distilled water and stained for $1 \mathrm{~min}$ in $0.1 \%$ aqueous solution of eosin Y. After short rinsing in distilled water the stained smears are rapidly dried. Another alternative depends on the combined use of the cytochemical reaction and the differential staining for live and dead spermatozoa. Under these circumstances a drop of $0.5 \%$ eosin solution is added at the end of the incubation, and then, $20 \mathrm{sec}$ later, the smears are prepared and rapidly dried.

\section{Demonstration of the succinate dehydrogenase activity}

The reaction for succinate dehydrogenase activity differs from the method described above in that to one drop of semen sample and Nitro BT solution (see above) a drop of $0.1 \mathrm{~m}$ sodium succinate is added.

\section{EXPLANATION OF PLATE 1}

Photomicrographs of bovine spermatozoa with different endogenous dehydrogenase activity. Active mitochondria appear as bright granules in the middle piece, due to high density of diformazan deposit, as observed in the phase microscope 'Anoptral' $\mathrm{C}$. Reichert Vienna.

Fig. 1. Three active (Class $1,+++$ ) and three inactive (Class $4,-$ ) spermatozoa.

FIG. 2. Three active (Class 2 and $3++,+$ ) and two inactive (Class $4,-$ ) spermatozoa.

FIG. 3. Spermatozoon with high density of active mitochondria (Class $1,+++$ ).

Frg. 4. Left, high activity (Class $1,+++$ ), right, low activity of mitochondrial sheath.

FIG. 5. Some segments of mitochondrial spiral inactive (Class $2,++$ ).

FIG. 6. Marked decline in mitochondrial sheath activity. 


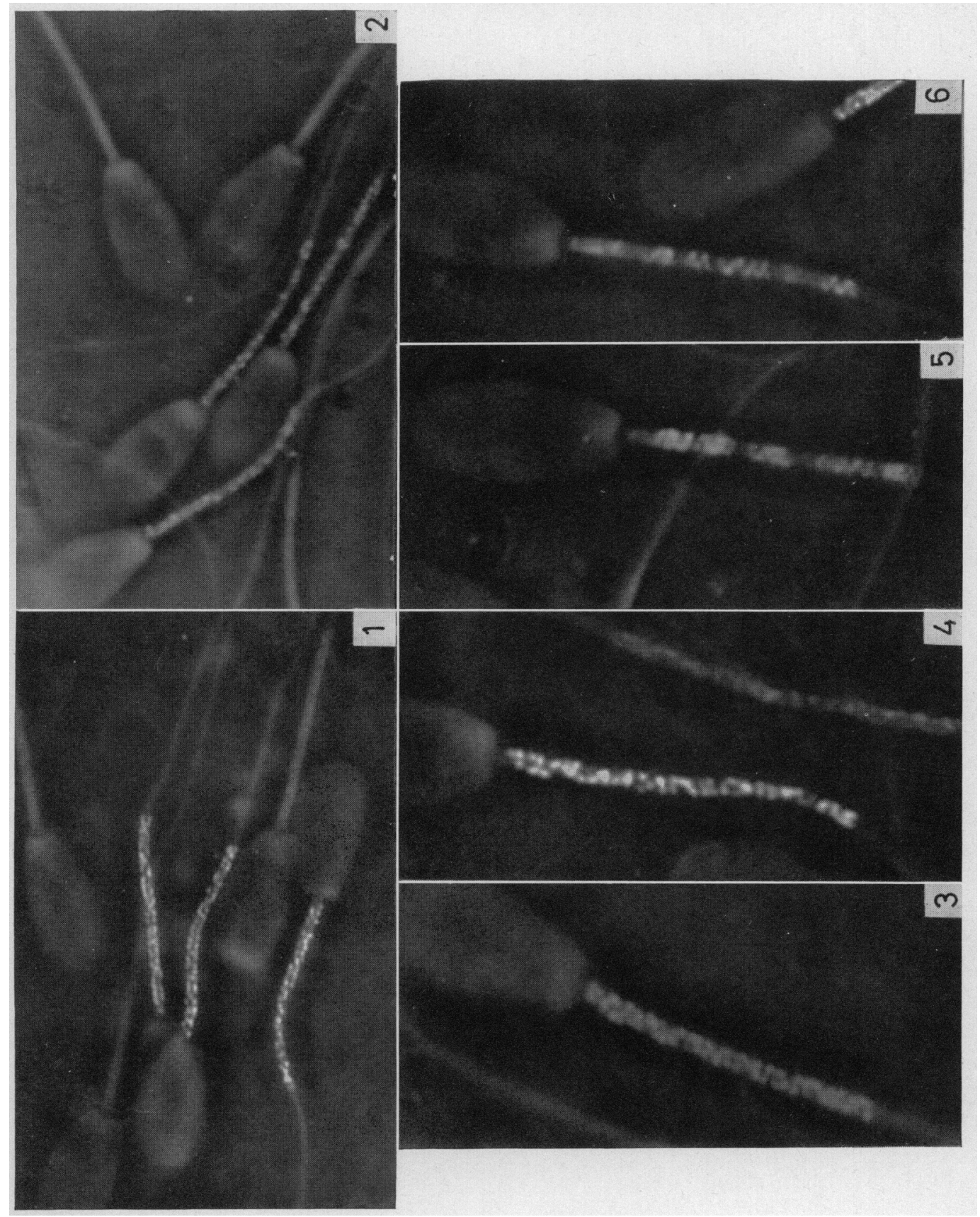


Evaluation of the dehydrogenase activity and classification of spermatozoa

Evaluation of the sperm smears is carried out like an evaluation of blood smears, that is by observing and classifying the spermatozoa which are present in individual fields under the microscope. With dense smears it is recommended to limit the field of view as much as possible. The smears are examined using a strong immersion objective, preferably of a phase-microscope, which offers more contrast because of the high density of diformazan deposits. One or two smears from each sample are evaluated as a rule, but a larger number of smears increases the accuracy of assessment. From 100 to 300 spermatozoa are counted on each smear.

The following scale for the classification of spermatozoa based on the appearance of their mitochondrial sheath, i.e. colour intensity, size and density of granules (mitochondria), has been adopted (see also Plate 1) in the present study: Class $1(+++)$ : Mitochondrial sheath exhibiting a high density of

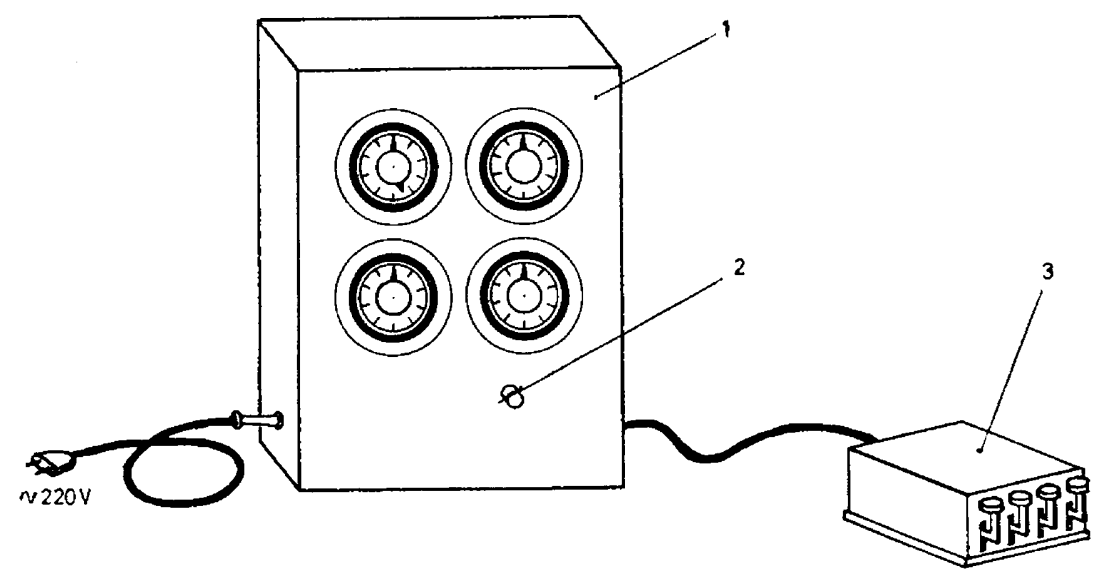

TeXT-FIG. 1. Registration apparatus for classification of spermatozoa. 1, Box with electromagnetic counters; 2 , button for resetting; 3 , box with four press-buttons for counting.

intensely stained, large-sized granules. Class $2(++)$ : Mitochondrial sheath with medium activity. Class $3(+)$ : Mitochondrial sheath with weak activity or only isolated active mitochondria; to the last class belong also those spermatozoa in which the middle piece shows only a diffuse reaction, with or without a trace of mitochondrial activity. Class $4(-)$ : Spermatozoa without any activity whatsoever.

For counting we used a specially constructed registration apparatus, which may be seen in Text-fig. 1. It consists of a box with four electromagnetic counters (type MO 1A, Microtechna, Prague) controlled by four press buttons on a small box placed close to the microscope. Registration of spermatozoa evaluated is made by means of the four press buttons; resetting can be done by rectifying the alternating current by means of the button on the main box. The classification apparatus may also be applied in other spermiological work. 
Estimation of sperm motility

Motility estimations, i.e. determinations of the percentage of motile and non-motile spermatozoa, were made on a microscope equipped with a thermostage set at $37^{\circ} \mathrm{C}$ and an orthoscopic ocular with a mask limiting the field of view.

\section{RESULTS}

The endogenous dehydrogenases activity of spermatozoa, i.e. as exhibited in the absence of extraneous substrates, is reflected in the formation of blue diformazan deposits, localized in the middle piece. These deposits indicate the position of the active individual mitochondria or of the mitochondrial sheath (Plate 1). The rate of the reaction in the mitochondria is related both to the staining intensity and to the size of the diformazan deposits. Very active mitochondria appear as intensely-stained, large-sized granules. On the other hand, mitochondria with low activity appear as weakly-stained, small-sized granules. It is of considerable interest to note that the mitochondrial sheath of an ejaculated spermatozoon is hardly ever uniformly stained throughout its whole length, and there exist some short segments corresponding to single mitochondria or whole mitochondrial groups which show little or no activity. With the natural ageing of spermatozoa the proportion of the 'inactive' segments increases.

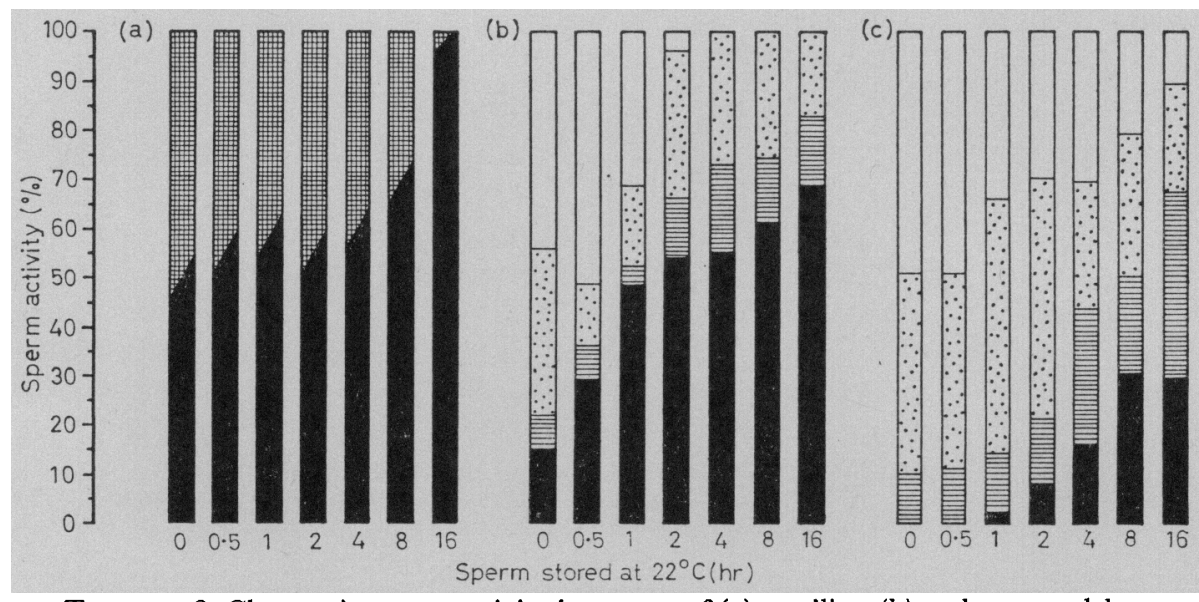

TeXT-FIG. 2. Ghanges in sperm activity in respect of (a) motility, (b) endogenous dehydrogenases and (c) succinate dehydrogenase, as observed in bovine sperm stored at room temperature. Motility expressed as percentage of motile spermatozoa (solid part of column, non-motile; shaded part, motile); dehydrogenase activity expressed as percentage of spermatozoa belonging to four classes: +++ , highest activity (open column); ++ , medium (stippled column); + , weak (shaded column); - , no activity (solid column).

The relationship between motility, endogenous dehydrogenase and succinate dehydrogenase activity is seen in Text-fig. 2. By comparing the percentage of motile spermatozoa with the level of endogenous dehydrogenase activity, it can be seen that these two types of sperm activity do not equal each other; the proportion of spermatozoa with dehydrogenase activity is generally higher than the proportion of motile ones. It would seem that some of the spermatozoa which have lost their motility are nevertheless able to preserve their endogenous 
dehydrogenase activity. When the endogenous activity is compared with that of succinate dehydrogenase, it becomes evident that the latter is of a higher order and persists for a much longer time. Thus it appears that the process of the spontaneous dying of spermatozoa begins with alterations and arrest of motility, which is then followed by cessation of endogenous dehydrogenase activity and leads finally to stoppage of succinate dehydrogenase activity.

The endogenous activity can be inhibited by protein-denaturing agents such as trichloracetic acid, surface active substances, heating, drying, and also by several enzyme inhibitors such as iodacetate, arsenic trioxide $\left(\mathrm{As}_{2} \mathrm{O}_{3}\right)$ and potassium cyanide.

\section{DISGUSSION}

The mechanism underlying the dehydrogenase reaction in the mitochondrial sheath is undoubtedly related to the ability of spermatozoa to make use of anaerobic and oxidative metabolic processes as a source of energy for sperm motility. Electron transfer, mediated by dehydrogenases which occurs during such processes, can be followed by means of various electron acceptors such as methylene blue, tellurite or tetrazolium salts. In the past methylene blue has been frequently employed in the 'methylene blue reduction test'. However, the application of that test to sperm evaluation is limited for several reasons, mainly because the reduction velocity (decolorization time) depends on the total activity of a large population of cells, including spermatozoa, micro-organisms and other cells, and also because of the possible presence of extracellular reducing substances in seminal plasma; moreover, the activity of the individual spermatozoa cannot be evaluated since the sperm cells differ from each other. In contrast to methylene blue, the tetrazolium salts which are both soluble and almost colourless, penetrate into the cells, and by accepting electrons are readily reduced to insoluble and intensely coloured formazans. This property of tetrazolium salts, chiefly of Nitro BT, is utilized for the histochemical demonstration of specific dehydrogenases and diaphorases in tissue sections (Nachlas, Walker \& Seligman, 1958; Scarpelli, Hess \& Pearse, 1958; Pearse, 1960), for the localization of some dehydrogenases in spermatozoa (Kothare \& de Souza, 1957; Restivo \& Reverberi, 1957; Nelson, 1959; Gupta \& Kamboj, 1962; Hrudka, 1963, 1964; Lojda \& Pařizek, 1963; Young \& Edwards, 1963; Balogh \& Cohen, 1964), as well as for the detection of endogenous dehydrogenases activity in the mitochondrial sheath of spermatozoa (Hrudka, 1964). Thus the mitochondrial localization of diformazan deposits is, in itself, indicative of the presence of the dehydrogenase activity. We believe that Nitro BT reduction is due to the interactions of intracellular dehydrogenases and endogenous substrates.

The reduction of tetrazolium salts to formazan also can be followed macroscopically by observing colour development during incubation. The staining intensity is related to the period of incubation and to the activity and density of the spermatozoa. By keeping the sperm concentration, temperature and incubation period constant, it is possible to assess the rate of the sperm reducing capacity by determining the intensity of colour. This has been also stated independently by Mohri (1957) and Blackshaw (1960). However, estimations based on a macroscopic colour test are unreliable, partly because of diffusion 
of reducing enzymes from mitochondria into the seminal plasma or incubating medium and partly also because some reduction may be caused by cells other than spermatozoa. In ageing sperm specimens, the increase in the number of micro-organisms may be an additional cause of marked reaction.

Typical localization and the relatively constant number of mitochondria in spermatozoa enable one to estimate the extent and intensity of endogenous dehydrogenase activity that is indicative of the actual catabolic processes in the living cell. Thus the method presents a new approach to the study of cell catabolism, which makes it possible to investigate the proportional distribution of activity within a given cell population, and to evaluate and compare ejaculates of the same individual or of different individuals. The method may be recommended for field use as a test for assessing sperm quality regardless of whether the semen is fresh or has been preserved with yolk or milk diluents. It can also be used for experimental purposes, for example in studying those environmental and experimental factors which affect sperm metabolism (Hrudka, 1965; Hrudka \& Horáková, 1965).

\section{ACKNOWLEDGMENTS}

I wish to thank Dr B. Večerek, Prague, Kateřinská 32, for synthesizing Nitro BT, Dr Ing. M. Rudič for the construction of the registration apparatus and Mrs P. Zambory for technical assistance.

\section{REFERENCES}

Balogh, K. JR. \& Cohen, R. B. (1964) A cytochemical technique for studying oxidative enzyme system of mammalian spermatozoa in semen smears. Fertil. Steril. 15, 35.

Blackshaw, A. W. (1960) Glycolysis and reducing activity of ram spermatozoa in phosphate-containing media. Aust. F. biol. Sci. 13, 371.

Gupta, B. L. \& KamBoJ, V. P. (1962) Histochemical localization of succinic dehydrogenase activity in the mitochondria of some invertebrate spermatozoa. Nature, Lond. 193, 788.

HrudKa, F. (1963) Cytochemická metóda na hodnotenie vitality spermií (preliminary report). Biológia, Bratisl. 18, 937.

Hrudka, F. (1964) Ủber die Dehydrogenaseaktivität und die endogene Reduction in Bullenspermien. Acta histochem. 19, 346.

HrudKa, F. (1965) Cytochemical study of the sperm endogenous reductases activity. I. Effect of washing upon the activity of endogenous reductases and succinodehydrogenase of bovine spermatozoa. Vet. med. Prague, $1,41$.

HrudKa, F. \& HoRÁkOvá, A. (1965) Cytochemical study of the sperm endogenous reductases activity. II. Effect of the osmotic gradient upon the activity of endogenous reductases and succinodehydrogenase of bovine spermatozoa. Vet. med. Prague, 2, 101.

Kothare, S. N. \& DE SouzA, E. J. (1957) A preliminary report on the cytochemical demonstration of succinic dehydrogenase pattern in human spermatozoa. Curr. Sci. 26, 355.

LOJDA, Z. \& PAŘ́zeK, J. (1963) Ontogenic development and topochemistry of testicular dehydrogenases with special reference to cadmium necrosis of the testis. Physiol. bohemoslov. 12, 512.

Mонri, H. (1957) Effect of malonate on oxygen uptake and dehydrogenase activity in sea-urchin spermatozoa. F. Fac. Sci., Tokyo, IV, 8, 65.

Nachlas, M. M., Walker, W. H. \& Seligman, A. M. (1958) J. biophys. biochem. Cytol. 4, 29. Gited in Pearse (1960).

NeLson, L. (1959) Cytochemical studies with electron microscope. II. Succinic dehydrogenase in rat spermatozoa. Exp. Cell Res. 16, 403.

Pearse, A. G. E. (1960) Histochemistry theoretical and applied. Churchill, London.

Restrvo, F. \& Reverberr, G. (1957) Richerche sugli spermi di Ciona e. di Pattela. Localizatione della citochromoxidase, della succino-deidrogenase e del glicogeno. Acta Embryol. Morph. exp. 1, 164.

Scarpelli, D. G., Hess, R. \& Pearse, A. G. E. (1958) 7. biophys. biochem. Cytol. 4, 747. Cited in Pearse (1960).

Young, W. R. \& Edwards, R. G. (1963) Ultraviolet microscopy and the detection of formazan deposits. J. Histochem. Cytochem. 11, 444. 